\title{
Early seizures indicate quality of perinatal care
}

\author{
R J DERHAM, T G MATTHEWS, AND T A CLARKE
}

Rotunda Hospital, Dublin, Ireland

SUMMARY An analysis of antepartum, intrapartum, and postpartum variables was performed in a retrospective controlled study of 34 normally formed term infants who had perinatal asphyxia and subsequently displayed generalised seizures within 48 hours of birth. The aim was to identify any association, firstly between these variables and seizures, and secondly between these variables and subsequent morbidity and mortality among the seizure group.

Maternal age greater than 35 years, duration of labour, meconium stained liquor, abnormal intrapartum fetal heart rate trace, and operative delivery were associated with seizures. A low Apgar score at five minutes, and intermittent positive pressure ventilation at birth of longer than 10 minutes were associated with subsequent morbidity and mortality. A striking relation between poor intrauterine growth and either death or handicap in the asphyxia group emphasised the value of growth measurements as a predictor of outcome. The overall incidence of seizures was 1.6 per 1000 term deliveries. There was a significant correlation between the seizure incidence and the intrapartum mortality rate. The incidence of seizures secondary to asphyxia in term infants, occurring less than 48 hours after delivery, may be a valuable index of the quality of perinatal care.

It has been established in recent years that most seizures in the first 48 hours are directly attributable to perinatal factors ${ }^{12}$ and that most seizures secondary to asphyxia occur during this time. It has also been established that the occurrence of generalised seizures has a high prognostic correlation with future morbidity and mortality. ${ }^{2-4} 7$ Asphyxia, however, remains a difficult concept to define accurately; allied to this is the lack of an agreed index of asphyxial damage and consequences for future morbidity and mortality. Most current indices of asphyxia (for example fetal distress, cord blood $\mathrm{pH}$, and Apgar score) have well documented limitations and have failed to reflect the quality of perinatal care. ${ }^{16-8}$

Dennis and Chalmers ${ }^{1}$ have suggested that to assess the quality of perinatal care a 'non-mortality' index is needed which: (i) can be unambiguously identified during the perinatal period; (ii) is known to be strongly related to later morbidity; and (iii) is likely to reflect the quality of care. They proposed that the incidence of seizures occurring in term infants within the first 48 hours would provide such an index. ${ }^{1}$ We agree with this and also believe that a specific group of infants with seizures secondary to asphyxia can be identified. This incidence, we propose, will provide an index, which we presently lack, of the quality of perinatal care.

\section{Patients and methods}

The study population included all live, inborn births at the Rotunda Hospital from 1 January 1979 to 31 December 1982. During this period 34 normally formed term (more than 37 weeks' gestation) infants, who had documented perinatal asphyxia, subsequently had generalised seizures within 48 hours of birth. Asphyxia was deemed to have occurred if the infant had an Apgar score less than 6 at either one or five minutes and also showed signs of cerebral dysfunction. ${ }^{9}$ Blood glucose, sodium, and calcium concentrations were determined for all infants, and where indicated investigations for sepsis, including a lumbar puncture, were undertaken to exclude other causes of seizures. Seizures were defined as generalised, tonic-clonic seizures and were easily identified by neonatal staff. Subtle seizures such as eye rolling or other doubtful seizures were not included. All infants were admitted to the neonatal intensive care unit directly from 
the labour ward, with the exception of one infant who died in the labour ward. All infants' seizures were observed by the attending neonatal house officer. Twelve infants died and in 10 of these necropsies were performed. Twenty surviving infants have been followed up and assessed developmentally at age 1 year. The two infants lost to follow up were normal at the time of discharge from the intensive care unit and at their six week follow up visit.

A control group (comprising term infants born immediately before and after a study group infant) of 68 infants was also selected. Various antenatal, intrapartum, and postpartum variables were compared between the groups. Any association between these variables and subsequent outcome was investigated. An abnormal outcome was defined as death or handicap. Handicap was defined as the presence of definite abnormal neurological signs such as cerebral palsy, mental retardation, or epilepsy. This was evident in seven of the eight surviving handicapped infants, the other infant having an isolated sensorineural hearing deficit. An acute asphyxial insult was deemed to have occurred if there was evidence of an abruptio placentae, prolapse of cord, a tonic uterine concentration, severe antepartum haemorrhage, eclamptic fit, or hypovolaemic maternal collapse.

Indications for intrapartum fetal monitoring were as follows: clinical fetal heart rate abnormalities, meconium stained liquor, antepartum haemorrhage, labour duration longer than 10 hours, the use of epidural anaesthesia, and any high risk pregnancy. An abnormal tracing was one in which any of the following was displayed: late or variable decelerations, base line tachycardia greater than 160 beats per minute, loss of beat to beat variability, the presence of a sinusoidal pattern, and a base line bradycardia less than 100 beats per minute.

Barbiturates were not given prophylactically during the study period. Statistical analysis was performed using Student's $t$ or Pearson's $\chi^{2}$ distribution tests of significance.

\section{Results}

During the four year study period a total of 21212 infants of more than 37 weeks' gestation were born in the Rotunda Hospital, giving a seizure incidence of 1.6 per 1000 (corrected for births of infants of more than 28 weeks' gestation=1.36 per 1000 ). Figure 1 shows some general perinatal statistics for this hospital since 1976 . The early neonatal seizure rate in term infants secondary to asphyxia has a significant correlation with the intrapartum mortality rate $(\mathrm{P}<0.05)$.

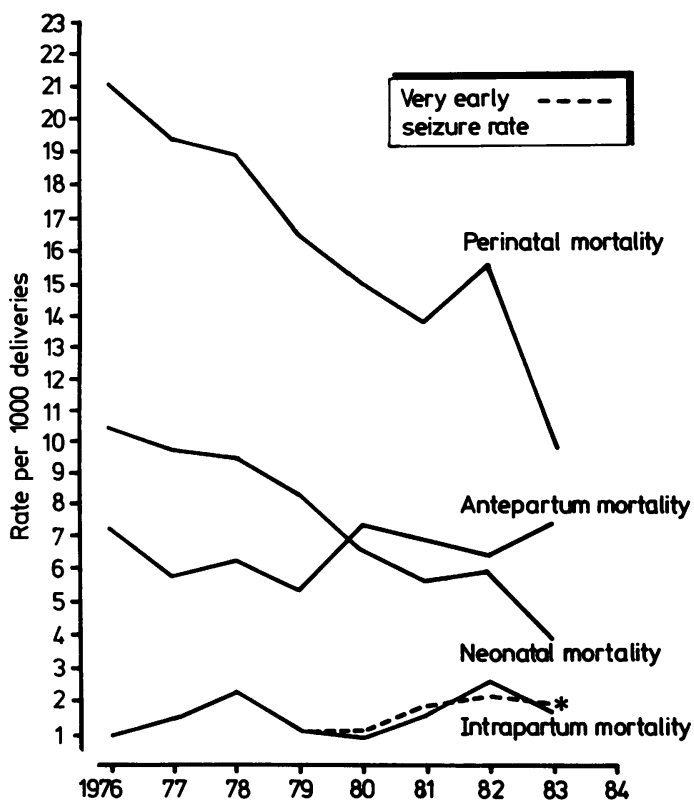

Fig. 1 Perinatal, neonatal, antepartum, and intrapartum mortality rates per 1000 deliveries greater than 28 weeks' gestation, together with very early seizure rate in term infants. (Rotunda Hospital 1976-83).

*Significant correlation with intrapartum mortality $\mathrm{P}<0.05$.

The antepartum variables analyses are shown in Table 1. There was a significant association between the occurrence of seizures and maternal age of 35 years and over. There was no difference for parity, mean gestational age, or in the number of infants

Table 1 Comparison of antepartum variables in 34 normally formed term babies who suffered seizures in the first 48 hours, and 68 controls

\begin{tabular}{|c|c|c|c|c|}
\hline \multirow[t]{2}{*}{ Antepartum variables } & \multicolumn{2}{|c|}{ Seizure group } & \multicolumn{2}{|c|}{ Control group } \\
\hline & No & $(\%)$ & No & \\
\hline \multicolumn{5}{|l|}{ Maternal age (yrs) } \\
\hline$<20$ & 3 & $(8 \cdot 8)$ & 6 & $(8 \cdot 8)$ \\
\hline $20-24$ & 7 & $(20 \cdot 6)$ & 14 & $(20 \cdot 6)$ \\
\hline $25-29$ & 14 & $(41 \cdot 2)$ & 24 & $(35 \cdot 3)$ \\
\hline $30-34$ & 3 & $(8 \cdot 8)$ & 20 & $(29.4)^{*}$ \\
\hline 35 & 7 & $(20 \cdot 6)$ & 4 & $(5.9)^{*}$ \\
\hline \multicolumn{5}{|l|}{ Gravid status } \\
\hline Primipara & 14 & $(41 \cdot 2)$ & 23 & $(33 \cdot 8)$ \\
\hline $2-4$ & 16 & $(47 \cdot 0)$ & 36 & $(52.9)$ \\
\hline $5-$ & 4 & $(11 \cdot 8)$ & 9 & $(13 \cdot 3)$ \\
\hline Smokers & 15 & $(44 \cdot 1)$ & 31 & $(45 \cdot 6)$ \\
\hline Antenatal complications & 11 & $(32 \cdot 4)$ & 9 & $(13 \cdot 2)$ \\
\hline Mean time of 1st visit (wks) & $17 \cdot 7$ & & $15 \cdot 7$ & \\
\hline Mean number of visits & $10 \cdot 2$ & & 9 & \\
\hline $\begin{array}{l}\text { Mean interval between last visit } \\
\text { and delivery (days) }\end{array}$ & $5 \cdot 2$ & & $5 \cdot 7$ & \\
\hline
\end{tabular}

${ }^{*} \mathrm{P}<0.05$. 
over 42 weeks' gestation. None of these variables had any bearing on subsequent outcome. There was no association with previous obstetric history.

When the intrapartum variables were looked at (Table 2) several differences emerged. More infants in the seizure group had meconium stained liquor, more had had fetal monitoring performed, and a significant number of these displayed an abnormal tracing. When analysed for poor outcome (that is death or handicap), a greater proportion of the seizure group who had abnormal intrapartum tracings had a normal outcome $(P<0 \cdot 01)$. This warrants further investigation with larger numbers.

Table 2 Comparison of intrapartum variables in 34 normally formed term babies who suffered seizures in the first 48 hours and 68 controls

\begin{tabular}{|c|c|c|c|c|}
\hline \multirow[t]{2}{*}{ Intrapartum variable } & \multicolumn{2}{|c|}{ Seizure group } & \multicolumn{2}{|c|}{ Control group } \\
\hline & No & $(\%)$ & & \\
\hline \multicolumn{5}{|l|}{ Onset of labour } \\
\hline Spontaneous & 28 & $(82 \cdot 4)$ & 59 & $(86 \cdot 8)$ \\
\hline Induction & 4 & $(11 \cdot 8)$ & 3 & $(4.4)$ \\
\hline Acceleration & 18 & $(52.9)$ & 24 & $(35 \cdot 3)$ \\
\hline Meconium liquor & 16 & $(47 \cdot 1)$ & $17^{*}$ & $(25 \cdot 0)$ \\
\hline Monitored & 26 & $(76 \cdot 5)$ & $30^{* *}$ & $(44.0)$ \\
\hline Abnormal monitor & 17 & & $9^{*}$ & \\
\hline Epidurals & 11 & $(32 \cdot 4)$ & 14 & $(20 \cdot 6)$ \\
\hline \multicolumn{5}{|l|}{ Pethidine } \\
\hline Total no & 13 & $(38 \cdot 2)$ & 26 & $(38 \cdot 2)$ \\
\hline$<2$ hours duration & 6 & $(17 \cdot 6)$ & 9 & $(13 \cdot 2)$ \\
\hline \multicolumn{5}{|l|}{ Mode of delivery } \\
\hline Normal vaginal & 3 & $(8 \cdot 8)$ & $44^{* * *}$ & $(64 \cdot 7)$ \\
\hline Breech & 4 & $(11 \cdot 8)$ & 3 & $(4 \cdot 4)$ \\
\hline Straight forceps & 9 & $(26 \cdot 5)$ & $6^{*}$ & $(8 \cdot 8)$ \\
\hline Rotation forceps & 0 & (Nil) & 3 & $(4 \cdot 4)$ \\
\hline Vacuum & 4 & $(11 \cdot 8)$ & 3 & $(4.4)$ \\
\hline Elective caesarean & 2 & $(5 \cdot 8)$ & 6 & $(8 \cdot 8)$ \\
\hline Emergency caesarean & 12 & $(35 \cdot 3)$ & $3^{* * *}$ & $(4 \cdot 4)$ \\
\hline Mean duration of labour (hrs) & $8 \cdot 3$ & & $6 \cdot 1^{*}$ & \\
\hline
\end{tabular}

${ }^{*} \mathrm{P}<0 \cdot 05,{ }^{* *} \mathrm{P}<0.01,{ }^{* * *} \mathrm{P}<0.001$
There was no association with epidural anaesthesia or the use of pethidine in labour. Mothers of infants in the seizure group had significantly longer labours.

When the mode of delivery was examined only $8.5 \%$ of the seizure group had a normal vaginal delivery. No association was found between intrapartum variables and subsequent abnormal outcome.

The postpartum variables analysed are shown in Table 3. The seizure group had lower Apgar scores at one and five minutes, a greater need for

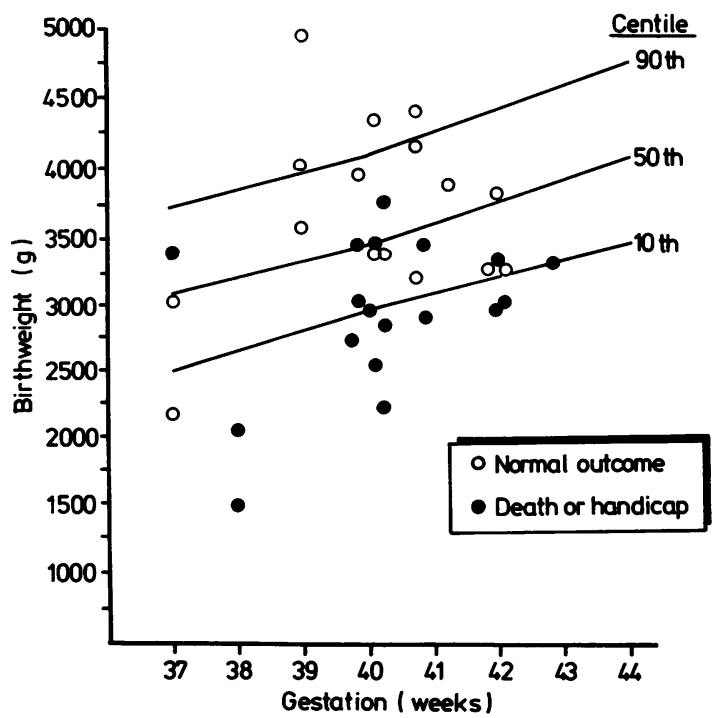

Fig. 2 Centile birthweights in relation to outcome in 34 term infants who suffered seizures in the first 48 hours of life.

Table 3 Comparison of postpartum variables between the groups and relation between these variables and subsequent normal or abnormal (death or handicap) outcome in the seizure group

\begin{tabular}{|c|c|c|c|c|c|c|}
\hline \multirow[t]{2}{*}{ Postpartum variables } & \multirow{2}{*}{\multicolumn{2}{|c|}{$\begin{array}{l}\text { Seizure group } \\
\text { No }(\%)\end{array}$}} & \multirow{2}{*}{\multicolumn{2}{|c|}{$\begin{array}{l}\text { Control group } \\
\text { No (\%) }\end{array}$}} & \multicolumn{2}{|c|}{$\begin{array}{l}\text { Relation between variables } \\
\text { and outcome in seizure group }\end{array}$} \\
\hline & & & & & $\begin{array}{l}\text { Normal } \\
\text { No }\end{array}$ & $\begin{array}{l}\text { Abnormal } \\
\text { No }\end{array}$ \\
\hline \multicolumn{7}{|l|}{ Sex } \\
\hline Boy & 19 & (56) & 34 & (50) & 6 & 12 \\
\hline Girl & 15 & (44) & 34 & $(50)$ & 7 & 9 \\
\hline Birthweight $<10$ th centile & 10 & (29) & 11 & (16) & 1 & $9^{*}$ \\
\hline Apgar score $\leqslant 5$ at $1 \mathrm{~min}$ & 31 & (91) & $11^{* * *}$ & (16) & 10 & 20 \\
\hline Apgar score $\leqslant 5$ at 5 mins & 20 & (59) & $0^{* * *}$ & Nil & 3 & $15^{* *}$ \\
\hline Intubated & 22 & (65) & $7^{* * *}$ & $(10)$ & 9 & 14 \\
\hline IPPV longer than 10 mins & 22 & (62) & $0^{* * *}$ & Nil & 0 & $14^{* * *}$ \\
\hline \multicolumn{7}{|l|}{ Overall outcome } \\
\hline Normal & 14 & $(41)$ & $67^{* * *}$ & (98) & & \\
\hline Abnormal & 20 & (59) & $1+* * *$ & (2) & & \\
\hline
\end{tabular}

${ }^{*} \mathrm{P}<0.05,{ }^{* *} \mathrm{P}<0.01,{ }^{* * *} \mathrm{P}<0.001$

†Died from sudden infant death syndrome. IPPV $=$ Intermittent positive pressure ventilation. 


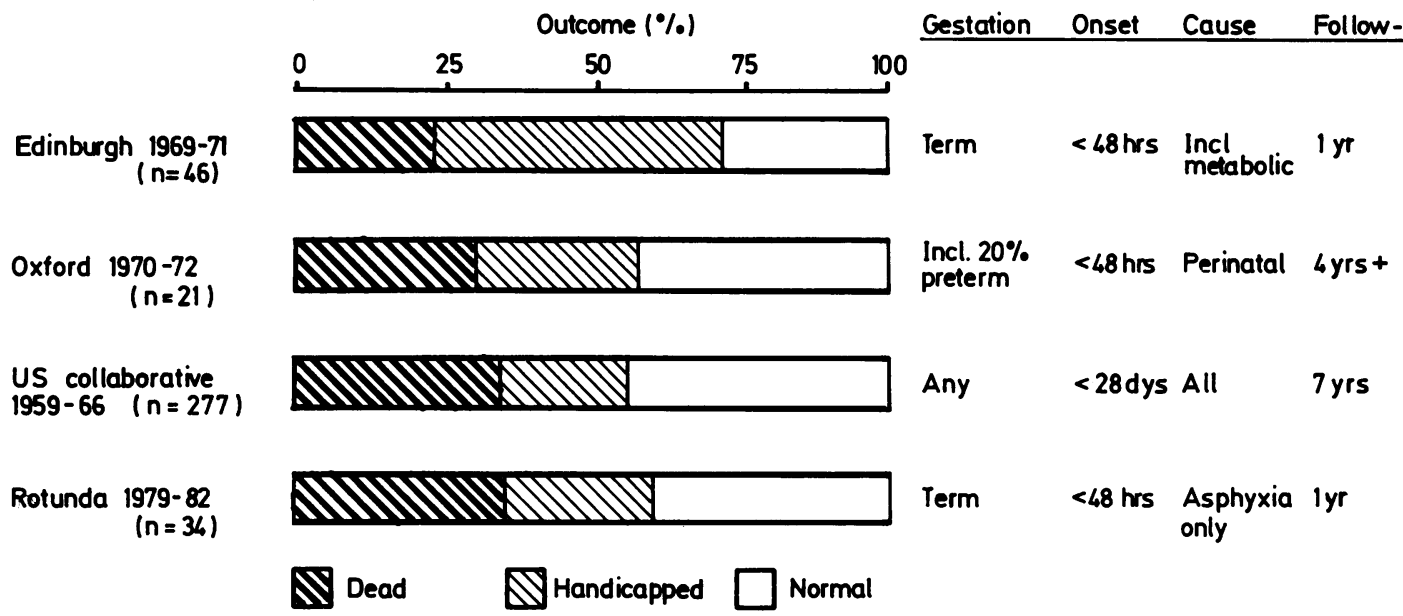

Fig. 3 Outcome after neonatal seizures.

intubation, and a longer period of intermittent positive pressure ventilation was required. Twenty nine per cent of the seizure group were below the 10th centile for weight, and Fig. 2 illustrates that most infants with a subsequent abnormal outcome were below the 50th centile. Weight less than the 10th centile, the Apgar score less than or equal to 5 at five minutes, intubation, and intermittent positive pressure ventilation for longer than 10 minutes were associated with a poor outcome in the seizure group. No seasonal variation was found. Figure 3 compares the outcome of our group with those of some other series. Twelve of our infants died, eight were handicapped, and 10 were normal at age 1 year. Two infants who were normal at 6 weeks of age were lost to follow up.

\section{Discussion}

The early neonatal seizure rate in term infants presented in this paper is a modification of a rate suggested by Dennis and Chalmers ${ }^{1}$ as a useful index to assess perinatal outcome. They highlighted the limitations of indices of perinatal care that are in current use such as the perinatal mortality rate.

Generalised tonic-clonic seizures occurring in term infants are readily identified by experienced neonatal personnel, and as an extreme manifestation of cerebral dysfunction allow for little observer error. When these occur less than 48 hours after birth it is relatively easy to assign a probable cause. Infants with asphyxial damage usually have other evidence of hypoxic-ischaemic injury such as prenatal fetal distress, poor condition at birth with the need for vigorous and prolonged resuscitation, and subsequent abnormal neurological behaviour. Causes of seizures that may be excluded readily by simple blood sampling include hypoglycaemia, hypocalcaemia, and hypo- and hypernatraemia. The exclusion of sepsis requires a full investigation including lumbar puncture and serology for congenital (viral and toxoplasma) infections. The advent of high resolution ultrasound makes the diagnosis of cerebral trauma and malformation relatively easy.

There is an unquestioned association between neonatal seizures and subsequent morbidity and mortality. By concentrating, however, on asphyxial seizures in term infants we remove the effect of gestation and of metabolic seizures, which have varying influences on subsequent outcome. It also provides us with a method of assessing the effect of various interventions in the perinatal period.

When factors that might predict the occurrence of seizures and subsequent outcome are analysed, the paucity of antenatal predictive factors is strikingly evident. The only significant variable isolated in our study was maternal age greater than 35 years; there was an equally significant reduction in the incidence of seizures in mothers aged 30 to 34 years old. The United States collaborative study based on data accumulated in the early 60 s found maternal age greater than 30 years to be significant. Traditionally used antenatal assessment of fetal well being, and perhaps more importantly assessment of the fetus's ability to withstand labour and delivery, are clearly wanting in their predictive effect. Present work on fetal assessment ${ }^{10}$ may provide the answer. 
In addition, many of the traditional methods of intrapartum fetal assessment are not sensitive enough to prevent asphyxial damage. This paper illustrates the fact that while meconium stained liquor was significantly associated with the seizure group, nearly $52 \%$ of these infants did not have meconium liquor yet suffered an asphyxial insult severe enough to cause seizures. The fact that the presence of meconium is more significantly associated with increasing gestational age than low apgar scores has already been noted by one of the authors $^{11}$ who questioned its value as a reliable index of fetal distress. When fetal heart monitoring in our study group is considered it is evident that despite clinical awareness severe asphyxia still occurred. We referred previously to a curious finding in our control group. Among the seizure group infants who had an abnormal tracing in labour, there was a higher percentage of normal outcomes. This may reflect a more rapid intervention when faced with a recognised, abnormal monitor pattern, highlighting the lack of sensitivity of fetal heart rate monitoring. The shortcomings of other intrapartum assessments have recently been reviewed. ${ }^{78}$ Of the 34 infants in the present study group, 26 had continuous fetal heart rate monitoring performed during labour, and this was abnormal in 17. This means that in 17 cases the fetal heart was either not monitored or considered normal during labour, In only two cases, however, was the quality of intrapartum care clearly deficient.

In Fig. 2 we illustrated the effect of centile weight on subsequent outcome. It re-emphasises the poor outcome of small for dates babies who suffer severe asphyxia, ${ }^{12}$ and underlines the value of including birthweight in any attempt to establish guidelines for house staff faced with the difficult problem of how long to persist with resuscitation in term infants with asphyxia. In this group, only one infant of the 11 with a birthweight below the 10th centile was normal at 1 year of age. Chronic asphyxia is usually associated with intrauterine growth retardation and similarly the greater proportion of hypoxic ischaemic encephalopathy is of a chronic nature. ${ }^{13}$ The structural damage associated with this chronic injury in term infants is usually manifest by the type of seizures we have described..$^{5}$ Acute hypoxicischaemic encephalopathy displays a different picture. ${ }^{13}$ This is an important point, as only two of our patients suffered an acutely definable asphyxial episode. It is possible there may be small, even recurrent episodes of an acute nature in what is essentially a chronic pathology. The assessment of this pathology and its importance for the well being of the fetus provides a major challenge for obstetrics in the future. A fully integrated assessment and management regimen of the kind currently being developed $^{10}$ may be the key.

The eventual outcome of normally formed term infants, who comprise the bulk of our workload, is directly related to their growth and condition at birth. Improvement in their prognosis lies not in the neonatal care that they receive, but in their antepartum and intrapartum assessment. An index that will measure the benefits of any future advances in this area is required. Presently used indices will not do so accurately enough and we propose the adoption of an index along the lines of that proposed by Dennis and Chalmers. We feel that it is possible to identify a group of term infants who have generalised seizures secondary to asphyxia within $\mathbf{4 8 \text { hours }}$ of birth, which we feel can provide a objective yardstick against which to assess future developments in perinatal care.

\section{References}

${ }^{1}$ Dennis J, Chalmers I. Very early neonatal seizure rate-a possible epidemiological indicator of the quality of perinatal care. Br J Obstet Gynaecol 1982;89:418-26.

2 Dennis J. Neonatal convulsions-aetiology, late neonatal status and long term outcome. Dev Med Child Neurol 1978;20:143-58.

${ }^{3}$ Lombroso CT. Neonatal seizures. In: Brown TB, Fellman RG, eds. Epilepsy: diagnosis and management. Boston: Little, Brown and Co, 1983.

4 de Souza SW, Richards B. Neurological sequelae in newborn babies after perinatal asphyxia. Arch Dis Child 1978;53:564-9.

${ }^{5}$ de Souza SW. Neurodevelopmental outcome after birth asphyxia. In: Chiswick ML, ed. Recent advances in perinatal medicine 1. Edinburgh: Churchill Livingstone, 1983.

6 Sykes GS, Molloy PM, Johnson P et al. Do Apgar scores indicate asphyxia. Lancet 1982;i:494-6.

${ }^{7}$ Lagercrantz H. Asphyxia and the apgar score. Lancet 1982;i:965.

8 Wood C. Care of the foetus during labour. In:Chiswick ML, ed. Recent advances in perinatal medicine 1. Edinburgh: Churchill Livingstone, 1983.

9 O'Brien N, Sheehan P, Barry C. A pilot study-cerebral dysfunction in the newborn. Irish Med J 1974;67:562-9.

${ }^{10}$ Manning FA, Morrison I, Lange IR, Harman CR. Antepartum evaluation of foetal health. In: Chiswick ML, ed. Recent advances in perinatal medicine. Edinburgh: Churchill Livingstone, 1983.

11 Matthews TG, Stack JC, Unwin A, et al. Is there a gestational age distribution in the passage of meconium.J Obstet Gynaecol 1983;4:23-4.

12 Alberman E, Benson J, McDonald A. Cerebral palsy and severe educational subnormality in low-birth weight children. Lancet 1982;i:606-8.

13 Volpe JJ. Perinatal hypoxic-ischaemic brain injury. Pediatr Clin North Am 1976;23:383-97.

Correspondence to $\operatorname{Dr} T$ G Matthews, Rotunda Hospital, Dublin 1, Ireland.

Received 2 April 1985 